\title{
Forschungsunterstützung an australischen Universitätsbibliotheken
}

\author{
Keller, Alice
}

\begin{abstract}
Forschungsunterstützung ist eine strategisch zunehmend wichtige Aufgabe an australischen Universitätsbibliotheken. Die Gründe hierfür sind in der stark leistungs- und wettbewerbsorientierten Forschungspolitik der Regierung sowie der Universitäten zu finden. Dieser Aufsatz beschreibt im Einzelnen die neuen forschungsunterstützenden Services, die von Bibliotheken während der letzten Jahre sukzessive auf- und ausgebaut wurden. Im Vordergrund stehen Services rund um Bibliometrie bzw. Research Impact, institutionelle Repositorien, spezifische Unterstützung von Forschungsstudenten sowie Forschungsdatenmanagement. Ein weiterer wichtiger Aspekt der Studie ist die organisatorische Verankerung dieser Aufgaben in der Bibliothek
\end{abstract}

DOI: https://doi.org/10.1515/bfp-2014-0060

Posted at the Zurich Open Repository and Archive, University of Zurich ZORA URL: https://doi.org/10.5167/uzh-154948

Journal Article

Published Version

Originally published at:

Keller, Alice (2014). Forschungsunterstützung an australischen Universitätsbibliotheken. Bibliothek Forschung und Praxis, 38(3):478-491.

DOI: https://doi.org/10.1515/bfp-2014-0060 
Alice Keller

\section{Forschungsunterstützung an australischen Universitätsbibliotheken}

Zusammenfassung: Forschungsunterstützung ist eine strategisch zunehmend wichtige Aufgabe an australischen Universitätsbibliotheken. Die Gründe hierfür sind in der stark leistungs- und wettbewerbsorientierten Forschungspolitik der Regierung sowie der Universitäten zu finden. Dieser Aufsatz beschreibt im Einzelnen die neuen forschungsunterstützenden Services, die von Bibliotheken während der letzten Jahre sukzessive auf- und ausgebaut wurden. Im Vordergrund stehen Services rund um Bibliometrie bzw. Research Impact, institutionelle Repositorien, spezifische Unterstützung von Forschungsstudenten sowie Forschungsdatenmanagement. Ein weiterer wichtiger Aspekt der Studie ist die organisatorische Verankerung dieser Aufgaben in der Bibliothek.

Schlüsselwörter: Australien; wissenschaftliches Bibliothekswesen; Forschungsunterstützung; Bibliometrie; Forschungsevaluation; Research Impact; institutionelle Repositorien; Open Access

\section{Research Support at Australian University Libraries}

Abstract: Research support is a strategically increasingly important role of Australian university libraries. The reasons for this trend can be found in the strictly performance-based research politics of the government and the individual universities. This essay describes in detail the new research support services which libraries have been developing and extending during the last years. The main focus is on services such as bibliometrics, institutional repositories, specific support of research students and research data management. A further significant aspect of the study regards how these services are organizationally embedded in the library.

Keywords: Australia; academic libraries; research support; bibliometrics; research evaluation; research impact; institutional repositories; open access

DOI 10.1515/bfp-2014-0060

Dr. Alice Keller: alice.keller@zb.uzh.ch
Inhalt

1 Forschung als strategische Priorität Australiens . 478

1.1 Bibliotheken als Partner in der Forschung . . . . 478

1.2 Forschungsfinanzierung in Australien . . . . . . 479

2 Forschungsunterstützende Services von Bibliotheken ..................480

2.1 Institutionelle Repositorien als Datenlieferant für HERDC und ERA . . . . . . . . . . . . . . . . . 480

2.2 Open Access in Australien . . . . . . . . . 481

2.3 Bibliometrie sowie Optimierung des Research Impacts .................... 482

2.4 Unterstützung von Forschungsstudenten . . . . . 484

2.5 Forschungsdatenmanagement . . . . . . . . . 484

3 Verankerung forschungsunterstützender Services in der Bibliotheken . . . . . . . . . . . . 486

3.1 Verschlankung des Angebotes für Studierende . . 487

3.2 Fokussierung der Tätigkeit von Liaison Librarians auf forschungsunterstützende Aufgaben und Bildung von fachbezogenen Teams zur Nutzung von Synergien................... 487

3.3 Schaffung neuer Positionen zur Forschungsunterstützung bzw. zur Koordination dieser Aufgaben . 488

4 Schlussfolgerung .............. 489

\section{Forschung als strategische Priorität Australiens}

\subsection{Bibliotheken als Partner in der Forschung}

"If the merit of universities is judged almost solely on research performance, what can academic libraries do to support their universities, and how are we addressing these new requirements?"1 Diese Frage stellt Rebecca Parker, Research Services Librarian, Swinburne University of Technology, und eröffnet hiermit eine Diskussion, an der sich gegenwärtig alle australischen Universitätsbibliotheken

1 Parker, R.: What the Library Did Next: Strengthening Our Visibility in Research Support. In: Paper presented at VALA 2012, Melbourne, Australia, February 6-9, 2012. Online zugänglich: http://www.vala. org.au/vala2012-proceedings/vala2012-session-1-parker. 
intensiv beteiligen. Die stark leistungsorientierte Forschungspolitik der Regierung wird auch vom australischen Bibliotheksverband CAUL (Council of Australian University Librarians) als wegweisend für die Fokussierung der Strategien auf die Bereiche eResearch, institutionelle Repositorien und Forschungskompetenz erachtet. ${ }^{2}$

In der Tat verstehen sich australische Universitätsbibliotheken als unverzichtbarer Partner in der Forschung. Hierbei sehen sie sich weniger als passive Weggefährten, sondern vielmehr als aktive Verbündete in der Herausforderung, die Forschungsleistung der eigenen Universität gezielt zu steigern. Nicht selten findet man in den Leitbildern der Bibliotheken Ziele wie „enhance research performance“ (Macquarie University Library) ${ }^{3}$ oder „make a critical contribution to the achievement of Monash's research strategies" (Monash University Library) ${ }^{4}$. Dieses gesunde Selbstbewusstsein der australischen Bibliothekare fand ich - als sehr nüchterne Europäerin - sowohl erfrischend als auch faszinierend.

Die Forschungsleistung ist ein aktuelles und politisch wichtiges Thema in Australien. Mit Sorge verfolgt man den globalen Wettlauf. „How could we possibly be in a country that does not have one university in the top 20 in the world?"5 bemerkte vor kurzem der australische Finanzminister Joe Hockey, schließlich profitiere die australische Forschung von guter Ressourcenlage und man sei durchaus in der Lage, die besten und intelligentesten Bewerber anzulocken. Als besonders erfolgversprechend erachtet man die medizinische und biomedizinische Forschung und möchte sich außerdem als High-Tech-Standort im pazifisch-asiatischen Raum profilieren. Die klare Fokussierung auf individuelle und institutionelle Forschungsleistung soll das Ergebnis Australiens in den globalen Rankings verbessern.

\subsection{Forschungsfinanzierung in Australien}

Gläser, Lange und Laudel bezeichnen das Universitätssystem Australiens als „eingliedrig aber stark stratifiziert“ . ${ }^{6}$ Die Universitäten sind weitgehend autonom und entschei-

2 CAUL - Research Program: http://www.caul.edu.au/caul-pro grams/research.

3 Dokument öffentlich nicht zugänglich.

4 http://www.monash.edu.au/library/about/us/mission.html.

5 Hare, J.: Reforms Will not Boost Rankings, The Australian (26 May 2014), http://www.theaustralian.com.au/higher-education/reformswill-not-boost-rankings/story-e6frgcjx-1226929068589.

6 Gläser, J.; Lange, S.; Laudel, G.: Auswirkungen der evaluationsbasierten Forschungsfinanzierung an Universitäten auf die Inhalte der Forschung. Australien und Deutschland im Vergleich. In: Wissenschaftsrecht 42(4) (2009) S. 329-352. den selbst über Studienangebot, innere Struktur und Haushalt. Diese Autonomie wird allerdings dadurch eingeschränkt, „dass die australische Bundesregierung die finanzielle Abhängigkeit der Universitäten von den Mitteln des Bundes immer wieder benutzt, um in diese zu intervenieren “7 . Dies gilt insbesondere für die Grundfinanzierung der Forschung, die vollständig über Leistungsindikatoren definiert wird. Diese Leistungsindikatoren errechnen sich aus Höhe der kompetitiven Drittmittel, Zahl der (schnellen) Abschlüsse von Forschungsstudenten, sowie Zahl und Qualität des Publikationsoutputs.

Dieser Ansatz führt natürlich zu einem starken Wettbewerbsverhältnis zwischen den einzelnen Universitäten, nicht zuletzt, weil die australischen Hochschulen ab einem bestimmten Punkt die Nachfrage nach Studienplätzen und entsprechend die Einnahmen aus der Lehre kaum beeinflussen oder steigern können. Hinzu kommt, dass die Reputation einer australischen Universität viel stärker vom Forschungserfolg als von den guten Leistungen im Studien- und Lehrbetrieb abhängt.

Für die Verteilung der Forschungsmittel sind die zwei Evaluationsrunden HERDC und ERA ausschlaggebend. Ziel dieser Evaluationen ist die gerechte und transparente Verteilung der so genannten Research Block Grants, also der staatlichen Grundfinanzierung für die Forschung. ${ }^{8}$

HERDC (Higher Education Research Data Collection) ist eine jährliche Erhebung. Im Bereich des Publikationsoutputs werden sämtliche Monographien, Beiträge in Büchern, Zeitschriftenaufsätze und Konferenzberichte gezählt (alle mit Peer-Review). Zu Prüfzwecken muss die Universität einfachen Zugang zu allen Metadaten und Volltexten bieten. In dieser Runde wird nur die Gesamtzahl an gültigen Publikationen erhoben, Impact Factor o.ä. fließen an dieser Stelle nicht in die Evaluation ein.

ERA (Excellence in Research for Australia) bietet eine viel differenziertere Auswertung und wird alle zwei bis drei Jahre durchgeführt. Hier sind nebst detaillierten bibliometrischen Kriterien auch Expertengutachten auf Fachgebietsebene von Bedeutung. In der Evaluation werden sämtliche Publikationen der letzten sechs Jahre, getrennt nach Fachgebieten, aufgenommen. ${ }^{9}$ Nebst den traditionellen Publikationsarten können in einzelnen Disziplinen auch kreative Werke und alternative Reputationswerte einfließen. Bei ERA ist auch ein internationales Benchmarking möglich.

7 Ebenda S. 332.

8 Block Grant bedeutet, dass die Mittel der Universität als Ganzes, also nicht nach Disziplinen verteilt, zugesprochen werden.

9 Allerdings benötigt eine Universität mindestens 30 Publikationen pro Fachgebiet, damit die Disziplin evaluiert wird. 
Wie oben erwähnt, stellt der Publikationsoutput in den Evaluationen nicht die einzige Messgröße dar. Von Bedeutung sind auch die Höhe der Drittmittel sowie die Zahl der Abschlüsse von Forschungsstudenten innerhalb einer vorgegebenen Frist. Als Forschungsstudenten gelten Teilnehmer in Master-Programmen mit Forschungsausrichtung sowie Doktoranden.

\section{Forschungsunterstützende Services von Bibliotheken}

Nachdem Ziellinie und Spielregeln so klar vorgegebenen sind, ergeben sich für Universitätsbibliotheken verschiedene Möglichkeiten, sich als Partner im Wettkampf zu positionieren und zu profilieren. Hierzu zählen Erfassung und Verwaltung von publikationsrelevanten Metadaten in institutionellen Repositorien, Unterstützung einzelner Forscher bei der Optimierung ihres Research Impacts sowie konkrete Hilfestellung für Forschungsstudenten zur Erreichung ihres Abschlusses. Die Förderung von Open Access und Forschungsdatenmanagement gelten ebenfalls als forschungsunterstützend, da beide sehr eng verwandt sind mit der nationalen und universitären Forschungspolitik.

\subsection{Institutionelle Repositorien als Datenlieferant für HERDC und ERA}

Australien hat ein sehr gut ausgebautes System an institutionellen Repositorien. Diese Repositorien sind oft unter der Bezeichnung „Research Online“ auf den Websites der Bibliotheken $\mathrm{zu}$ finden. Zur Startfinanzierung standen zentrale Mittel zur Verfügung; danach gingen die Repositorien in den Verantwortungsbereich der Universitäten bzw. Bibliotheken über. Die Liste der Australian Open Access Support Group (AOASG) verzeichnet gegenwärtig 51 Repositorien an Universitäten und anderen Forschungseinrichtungen. ${ }^{10}$ Diese Repositorien spielen eine zentrale Rolle in der australischen Forschungsevaluation sowie in der nationalen Open-Access-Politik.

Eine gute Übersicht über den Inhalt eines Repositoriums bietet exemplarisch das QUT-ePrints-Archiv der Queensland University of Technology (vgl. Tab. 1). Auffallend ist die hohe absolute Zahl an Zeitschriftenaufsätzen

10 Australian OA Repositories: http://aoasg.org.au/open-access-re positories-at-australian-institutions/. Diese Website dokumentiert auch das darunterliegende Softwaresystem: Am stärksten verbreitet sind DSpace (13), Vital (10) und DigiTool (7) (Stand Mai 2014).
(26 744) und Konferenzbeiträgen (12 182), wobei ein großer Prozentsatz dieser Dokumente weder als Volltext noch als Open Access zugänglich ist. Dies erklärt sich daraus, dass Repositorien als Metadatenlieferant für die oben genannten Evaluationen HERDC und ERA möglichst vollständig und aktuell gepflegt werden müssen. Wo eine Diskrepanz zwischen Volltext und Open Access erkennbar ist, bleibt der Volltext für externe Nutzer langfristig oder solange gesperrt, bis eine Embargofrist abgelaufen ist.

Tab. 1: Inhalt des institutionellen Repositoriums QUT ePrints nach Dokumententyp (Stand Mai 2014) ${ }^{11}$

\begin{tabular}{lrrr}
\hline Item Type & Works & Full-text & Open Access \\
\hline Book & 1082 & $26 \%$ & $17 \%$ \\
\hline Book Chapter & 4042 & $43 \%$ & $11 \%$ \\
\hline Conference Item & 1206 & $71 \%$ & $66 \%$ \\
\hline Conference Paper & 12182 & $71 \%$ & $66 \%$ \\
\hline Creative Work & 560 & $75 \%$ & $63 \%$ \\
\hline Journal Article & 26744 & $47 \%$ & $40 \%$ \\
\hline Other & 660 & $84 \%$ & $80 \%$ \\
\hline QUT Thesis & 4013 & $66 \%$ & $65 \%$ \\
\hline Reference Entry & 65 & $37 \%$ & $9 \%$ \\
\hline Report & 964 & $83 \%$ & $79 \%$ \\
\hline Review & 301 & $45 \%$ & $35 \%$ \\
\hline Thesis & 130 & $72 \%$ & $65 \%$ \\
\hline Working Paper & 341 & $96 \%$ & $93 \%$ \\
\hline Total & 52290 & $56,9 \%$ & $48,3 \%$ \\
\hline
\end{tabular}

Zur Erreichung optimaler Resultate in den nationalen Evaluationsrunden HERDC und ERA existiert eine enge Zusammenarbeit zwischen den Research Offices der Universität ${ }^{12}$, den Bibliotheken und den Fakultäten. Die Universitätsbibliothek übernimmt in der Regel die Erfassung und Prüfung der Metadaten. (Diese wichtige Arbeit den Autoren zu überlassen, führt erfahrungsgemäß zu allzu unzuverlässigen und lückenhaften Resultaten.) Bibliothekare „grasen“ hierzu systematisch große bibliographische Datenbanken ab und suchen gezielt nach Publikationen eigener Wissen-

11 QUT ePrints Collection Breakdown: http://eprints.qut.edu.au/sta tistics/collection_statistics.

12 Alle australischen Universitäten verfügen über ein zentrales Research Office, das als Zentraleinrichtung für die Forschung fungiert. Einerseits unterstützt die Einrichtung die Wissenschaftler bei der Fördermittelakquise, andererseits koordiniert es die Vorbereitungen und Erhebungen für die Forschungsevaluationen ERA und HERDC. 
schaftler; wo erlaubt, speichern sie auch die Volltexte ab, oder fragen die Autoren nach Preprints.

Diese zentrale Bedeutung von institutionellen Repositorien in der Forschungsevaluation bringt für die Bibliothek allerdings nicht nur Vorteile. Michael Organ merkt an, dass insbesondere für die Aufbereitung der ERA-Daten so viele personelle Kapazitäten gebunden sind, dass andere wichtige Aufgaben eines Repositorium-Teams, wie beispielsweise Förderung von Open Access oder Aufnahme anderer Dokumententypen, zu kurz kommen. ${ }^{13}$ An einigen, aber nicht allen Universitäten, wurde der Bibliothek für die Vorbereitung von ERA eine zusätzliche Stelle zugesprochen. Organs größte Sorge gilt allerdings nicht den personellen Kapazitäten, sondern der Natur des Repositoriums: Die Einbindung des Repositoriums in den Forschungsevaluationsprozess hat dazu geführt, dass ein Großteil des nachgewiesenen Materials nicht frei zugänglich ist und somit dem ursprünglichen Open-Access-Gedanken widerspricht. Zusammenfassend meint er: „ERA was for many repositories, a dead weight. “14

Die Veränderung der Zwecks eines Repositoriums vom Open-Access-Portal hin zum Komplettnachweis für die Forschungsevaluation kann allerdings auch positiv gewertet werden: Die Universität Wollongong spricht von einer gewünschten „Umnutzung“ des Repositoriums als Plattform für den lückenlosen Nachweis aller universitätseigenen Publikationen. ${ }^{15}$ Um diesen Konflikt zwischen Datenlieferant für die Forschungsevaluation und Open Access zu lösen, hat die Universität Sydney zwei Repositorien aufgesetzt: Sydney Research Online für den Publikationsnachweis für HERDC und ERA und Sydney eScholarship Repository als Open-Access-Plattform.

Institutionelle Repositorien dienen allerdings nicht nur der Universität, sondern auch dem einzelnen Wissenschaftler als bibliographisch zuverlässiges Nachweisinstrument. Parker sieht das Repositorium als ausgezeichnetes Beispiel einer Bibliotheksservices, das sowohl dem Forscher als auch der Universität großen Nutzen bringt. ${ }^{16}$ Das Ziel der Bibliotheken ist es, diese Repositorien noch

13 Organ, Michael K.: Leveraging Research Quality Assessment Exercises to Increase Repository Content-an Australian Case Study. In: Paper presented at CAIRSS Community Day. Melbourne 2010. Online zugänglich: http://ro.uow.edu.au/cgi/viewcontent.cgi?article=1259\& context=asdpapers.

14 Ebenda S. 4.

15 Vgl. hierzu Daly, R.; McIntosh, L.M.: Heresy or Innovation? Transforming Culture and Service for Impact. In: THETA: The Higher Education Technology Agenda. University of Tasmania, Australia 2013. Hier zit. S. 6. Online zugänglich: http://ro.uow.edu.au/asdpapers/40 $6 /$.

16 Vgl. Parker (Anm. 1). nützlicher zu machen und stärker in den Evaluationsprozess einzubinden bzw. als persönlichen Reputationsspiegel einzelner Forscher zu nutzen, z.B. durch das Ausweisen von Downloadzahlen oder durch die Integration szientometrischer Werte aus anderen Quellen (z.B. Thomson Reuter Web of Science Citation Count, Scopus Citation Count, Altmetrics). Der Einsatz solcher Zusatzinformationen wird beispielsweise im institutionellen Repositorium der Universität Queensland (UQ eSpace) gezeigt. ${ }^{17}$

\subsection{Open Access in Australien}

Trotz der Befürchtung, dass Repositorien nicht gleichbedeutend mit Open Access sind, nehmen sie in der OpenAccess-Politik Australiens eine zentrale Stelle ein. In Bezug auf die Veröffentlichung von Publikationen, die aus zentral finanzierten Forschungsprojekten hervorgehen, befürwortet Australien klar den „Grünen Weg“, also die Parallel- oder Zweitveröffentlichung in einem Repositorium. So haben die zwei wichtigsten Forschungsförderer Australian Research Council (ARC) und National Health and Medical Research Council (NHMRC) folgendes Mandat für Publikationen verabschiedet:

- Die Metadaten der Publikation müssen sofort nach Annahme zur Veröffentlichung in einem institutionellen Repositorium hinterlegt werden.

- Die Volltexte der Publikation sollen möglichst rasch nach Erscheinen in einem institutionellen Repositorium abgelegt werden. Die öffentliche Zugänglichkeit kann allerdings zeitlich verzögert erfolgen; zulässig ist eine Embargofrist von maximal 12 Monaten (also „Grüner Weg").

- Lässt der Verlag keine öffentliche Zugänglichkeit nach 12 Monaten zu, muss dies gegenüber den Forschungsförderern explizit erwähnt werden.

- Alternativ zur Ablage des Volltextes im eigenen institutionellen Repositorium ist auch der Zugang über ein Fachrepositorium oder den Verlag („Goldener Weg“) möglich. In diesen Fällen wird eine Verknüpfung zu den Metadaten im institutionellen Repositorium gefordert.

Wie oben erwähnt, unterstützt die Open-Access-Politik Australiens den „Grünen Weg“, also die Parallelveröffentlichung in einem Repositorium. Für die Bezahlung von Autorengebühren (Article Processing Charges, APCs) - al-

17 UQ eSpace: http://espace.library.uq.edu.au/ (Tipp: Suche nach „Hubert Chanson“ als Autor). 
so zur Finanzierung des „Goldenen Weges“ - besteht seitens der Regierung keine Erwartung, dass die Forschungsförderer zusätzliche Mittel bereitstellen. Dennoch wird der „Goldene Weg“ als möglicher Publikationsmodus für Open Access akzeptiert und manche Universitätsdepartments stellen hierfür auch Mittel zur Verfügung, da sie sich hierdurch bessere Sichtbarkeit erhoffen.

Die Open-Access-Strategie von ARC und NHMRC wird in Abb. 1 dargestellt. Hier erkennt man auch klar, dass sich die Regelung Australiens sich nicht nur auf Zeitschriftenaufsätze, sondern auch auf Buchkapitel oder ganze Buchpublikationen bezieht.

\section{ARC \& NHMRC policy compliance flowchart by an institution}

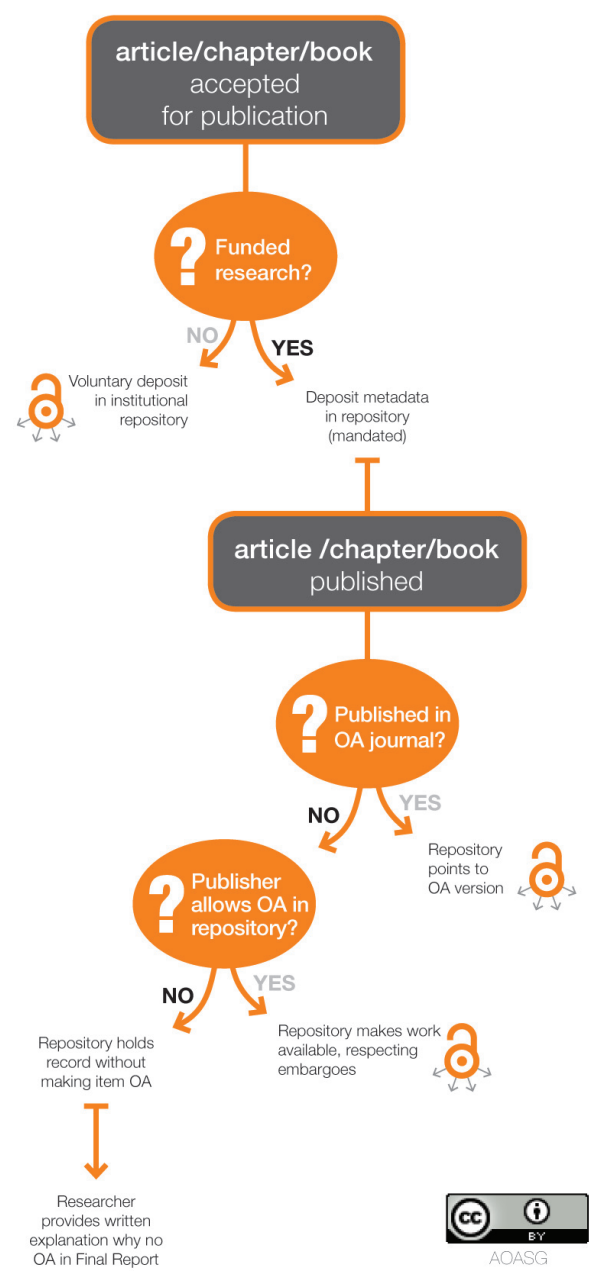

Abb. 1: Open-Access-Strategie von ARC und NHMRC (Quelle: AOASG: Policy Compliance Decision Tree18)

18 AOASG. Policy Compliance Decision Tree: http://aoasg.org.au/re sources/policy-compliance-decision-tree/.
Die Rolle der Bibliotheken im Bereich Open Access ist allerdings nicht nur auf den Aufbau und Betrieb von Repositorien beschränkt. Eine Bibliothek kann des Weiteren auch Beratungsdienste oder technisch-verlegerischen Support leisten. $\mathrm{Zu}$ den Beratungsleistungen zählt Zhao folgende Services, die sie unter dem Sammelbegriff „Scholarly Publishing Literacy“ zusammenfasst: ${ }^{19}$

- Aufklärung der Wissenschaftler zur Bedeutung und den verschiedenen Varianten von Open Access.

- Unterstützung der Wissenschaftler bei der Ermittlung möglicher Open-Access-Zeitschriften. Immer wichtiger wird hier die Prüfung der Qualität und Seriosität einzelner zahlpflichtiger Anbieter.

- Hilfestellung beim Verständnis der Autorenrechte sowie den Möglichkeiten einer Veröffentlichung unter Creative Commons Lizenz.

Der technisch-verlegerische Support bezieht sich hauptsächlich auf das Angebot geeigneter IT-Infrastruktur, z.B. Open-Journals-Software, DOI-Vergabe, Beratung von Herausgebern bei Urheberrechtsfragen und Autorenrichtlinien etc.

\subsection{Bibliometrie sowie Optimierung des Research Impacts}

Die zentrale Bedeutung von HERDC und ERA führt dazu, dass jedes Department, jedes Institut und jeder Wissenschaftler gut beraten ist, seine Publikationstätigkeit strategisch klug zu planen und szientometrische Analysen zu verstehen. Bei der Evaluation des Publikationsoutputs zählt, wie oben erwähnt, einerseits die absolute Zahl an Veröffentlichungen mit Peer Review und andererseits die Qualität der Publikationen. Zum Verständnis dieser Parameter sowie den verschiedenen Möglichkeiten zur Optimierung dieser Werte kann die Bibliothek auf verschiedenen Ebenen wertvolle Hilfestellung bieten. Dieses Serviceportfolio wird gewöhnlich unter dem Begriff „Research Impact“ zusammengefasst und gehört inzwischen zum Standardangebot einer australischen Universitätsbibliothek. Auf den Websites der Bibliotheken findet man zahlreiche und umfassende Informationen und Hilfestellungen zum Thema.

Hierbei darf Research Impact nicht lediglich auf die Messung des $h$-Indexes beschränkt werden. Die Bibliothek

19 Zhao, L.: Riding the Wave of Open Access: Providing Library Research Support for Scholarly Publishing Literacy. In: Australian Academic \& Research Libraries 45(1) (2014) S. 3-18 (hier zit. S. 13). 
versucht vielmehr, das Thema ganzheitlich anzugehen und zu erklären, wie die verschiedenen Elemente oder Phasen des Publikationszyklus ineinander greifen und so den Research Impact beeinflussen. $\mathrm{Zu}$ den Elementen oder Phasen dieses Zyklus gehören die Entwicklung einer geeigneten Publikationsstrategie, die professionelle Verwaltung der eigenen Publikationen, die Pflege des Autorenprofils (z. B. ORCID, Researcher-ID, Google Scholar ID), die Impact-Messung sowie die Selbstdarstellung der eigenen Ergebnisse (vgl. Abb. 2).

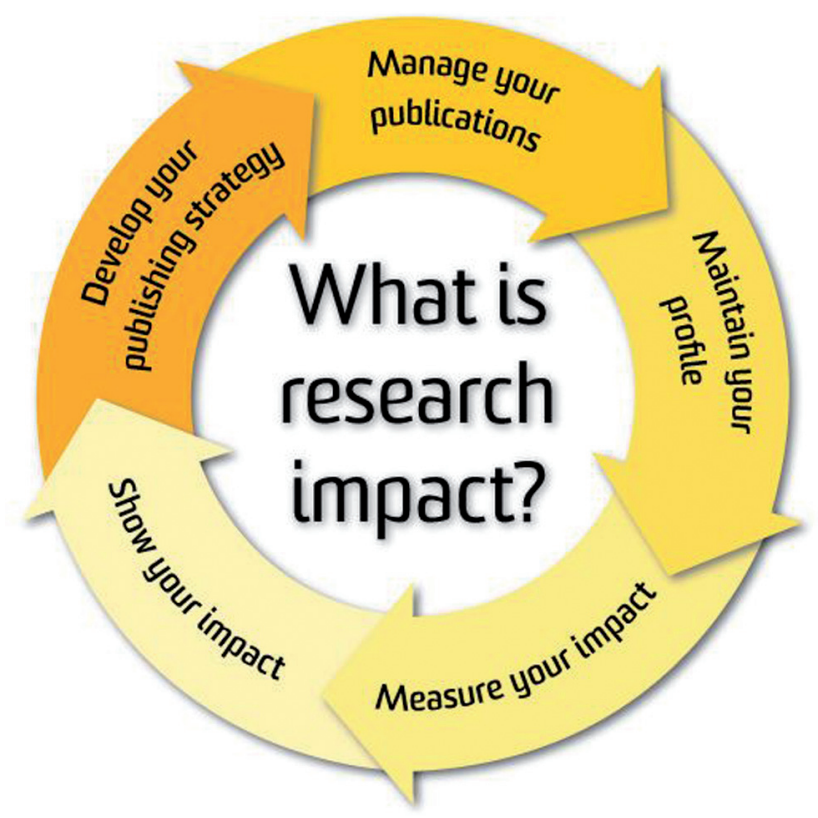

Abb. 2: Beispiel der Darstellung des Research Impact Cycle (Quelle: UNSW Research Impact Guide ${ }^{20}$ )

Die meisten Bibliotheken bieten für alle Phasen dieses Publikationszyklus umfassende Hilfestellung an. Hierzu gehören Online-Hilfen, Beratungs- und Schulungsangebote, Zugriff auf die benötigten Datenbanken (z.B. Web of Science, Scopus, JCR) und Tools (z. B. das Literaturverwaltungsprogramm Endnote). Diese Services werden in der Regel über einen themenspezifischen LibGuide angeboten. ${ }^{21}$ Nachfolgendes Schema zeigt den Inhalt des LibGuides zum Thema Research Impact an der University of New

20 UNSW Research Impact Guide: http://subjectguides.library.uns w.edu.au/researchimpact.

21 LibGuides ist ein Content-Management-System der Firma SpringShare, das von Bibliotheken weltweit genutzt wird. In Australien werden an fast allen Bibliotheken Fachinformationen über LibGuides angeboten. Diese lassen sich sehr einfach in Websites von Bibliotheken integrieren und weisen einen einheitlichen Aufbau auf.
South Wales (UNSW), entsprechend der Gliederung in Abb. $2 .^{22}$

\section{UNSW Library - Subject Guides - Research Impact Guide}

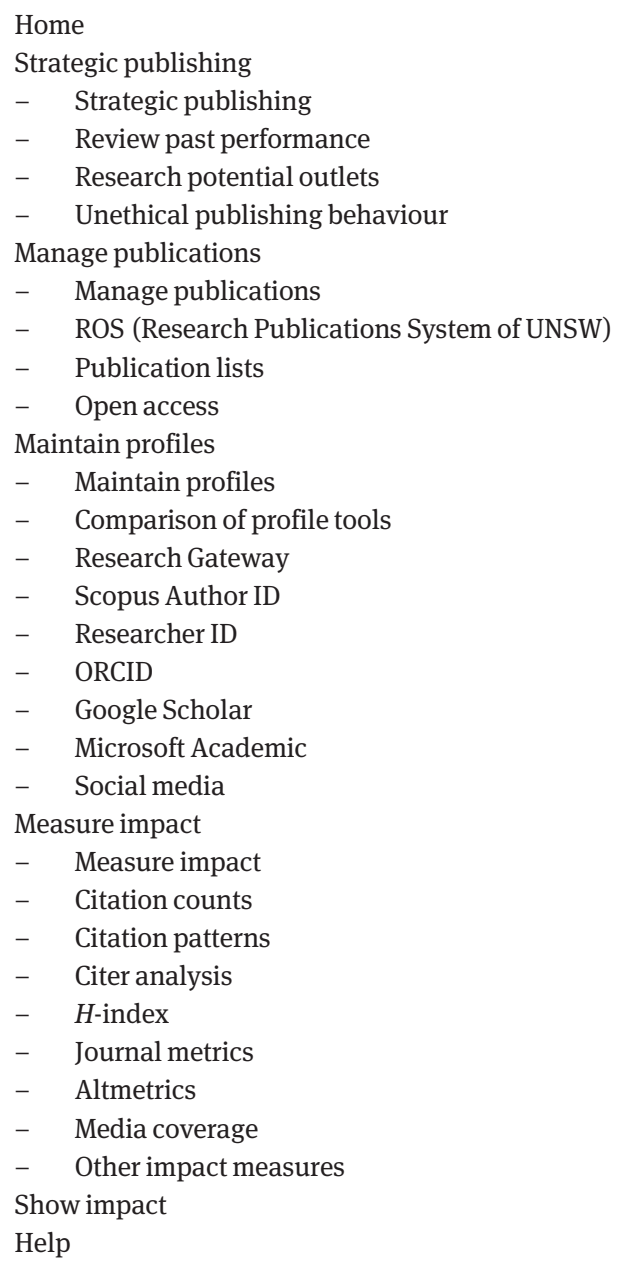

Anfangs boten manche Bibliotheken auch an, bibliometrische Analysen im Auftrag durchzuführen ${ }^{23}$, aber inzwischen ist klar geworden, dass die Bibliotheken ressourcenmäßig nicht in der Lage sind, einen flächendeckenden Service für alle Forscher anzubieten. Stattdessen konzentriert man sich auf gute Online-Hilfestellung, Schulungsund Beratungsdienste und bietet vielerorts maßgeschneiderte Expertenberichte für übergeordnete Fragestellungen

Beispiele: La Trobe: http://latrobe.libguides.com/; James Cook htt p://libguides.jcu.edu.au/.

22 UNSW Research Impact Guide: http://subjectguides.library.uns w.edu.au/content.php?pid=491680\&sid=4110927.

23 Vgl. hierzu beispielsweise: Drummond, R.; Wartho, R.: RIMS: The Research Impact. Measurement Service at the University of New South Wales. In: Australian Academic \& Research Libraries 40(2) (2009) S. 76-87. 
oder größere Forschungsanträge an. Gespräche mit Bibliothekaren zeigen, dass Forscher in naturwissenschaftlichmedizinischen Disziplinen in der Regel gut mit dem bibliometrischen Instrumentarium zurechtkommen. Hilfestellung benötigt vor allem Nachwuchswissenschaftler sowie Forscher in den Geistes- und Sozialwissenschaften. Bei letzteren versuchen die Bibliothekare auch auf die Einsatzmöglichkeiten von Altmetrics oder anderen Reputationswerten hinzuweisen.

Das Angebot an Research Impact Services stellt viele wissenschaftliche Bibliothekare vor neue persönliche Herausforderungen hinsichtlich szientometrischer Kenntnisse, Datenverarbeitung und Systemkenntnisse. Mary Anne Kennan, Charles Sturt University, weist in einem Vortrag darauf hin, dass diese Fähigkeiten leider bisher noch nicht Teil des regulären Lehrplans eines Bibliotheksstudiums sind. Die notwendigen Kenntnisse werden in der Regel im Beruf erworben; von Bedeutung sind hier auch produktbezogene Schulungen und Präsentationen kommerzieller Anbieter. Mangelhafte Kenntnisse und Fähigkeiten sowie fehlendes Selbstvertrauen der Bibliothekare stellen laut Corrall, Kennan und Afzal die größte Hürde bei der Entwicklung bzw. beim Ausbau bibliometrischer Services dar. ${ }^{24}$

\subsection{Unterstützung von Forschungsstudenten}

Die gezielte Unterstützung von Forschungsstudenten ist aus Sicht australischer Universitäten - und somit auch der Bibliotheken - sinnvoll und lohnenswert, da eine mögliche hohe Anzahl an HDR-Abschlüssen innerhalb einer vorgegebenen Frist finanzielle Vorteile bringt. HDR steht für „Higher Degree Research“ und bezieht sich auf Masteroder Doktorandenstudiengänge mit Schwerpunkt in der Forschung.

$\mathrm{Zu}$ diesen spezifischen Dienstleistungen der Bibliotheken für HDR-Studenten gehören maßgeschneiderte Einführungsseminare zur Unterstützung von Forschungsarbeiten, Hilfestellung bei der Literatursuche sowie Beratung hinsichtlich Dokumenten- und Datenverwaltung. Selbstverständlich bietet die Bibliothek auch Arbeitsplätze und Gruppenarbeitsräume - oft räumlich getrennt von den Studierenden unterer Semester - an. ${ }^{25}$ Bibliothekare an der Australian Catholic University erklärten mir, dass sie sämt-

24 Corrall, S.; Kennan, M.A.; Afzal, W.: Bibliometrics and Research Data Management Services: Emerging Trends in Library Support for Research. In: Library Trends 61(3) (2013) S. 636-674. Hier S. 660.

25 Vgl. hierzu beispielsweise: Richardson, J. et al.: Library Research Support in Queensland: A Survey. In: Australian Academic \& Research Libraries 43(4) (2012) S. 258-277, hier zit. S. 262. liche HDR-Studenten mit einer persönlichen Email begrüßten und ihnen einen Gesprächstermin mit ihrem Liaison Librarian anboten.

Verschiedene Studien zu den Informationsbedürfnissen von Forschungsstudenten weisen darauf hin, dass Nachwuchswissenschaftler besondere Erfordernisse in den Bereichen fachspezifischer Recherchen, Alert-Services (um auf dem Laufenden zu bleiben), Evaluation geeigneter Zeitschriften für die Publikation eigener Resultate und Datenmanagement haben. ${ }^{26}$ Die Technische Universität Queensland hat für diese Studentengruppe ein eigenes OnlineSchulungsprogramm namens „Advanced Information Research Skills“" entwickelt, das im Lehrplan integriert ist. ${ }^{27}$

\subsection{Forschungsdatenmanagement}

Forschungsdatenmanagement stellt im Moment ein sehr aktuelles Gesprächsthema zwischen australischen Bibliothekaren dar. Man vergleicht gegenseitig, wie die Rollenverteilung zwischen Bibliothek, IT-Diensten und Research Office aussieht, wie weit fortgeschritten die Datenmanagementpläne der verschiedenen Universitäten sind, welche Projekte am Laufen sind und wie hoch entwickelt das „Daten-Bewusstsein“ der Wissenschaftler ist.

Der Australian National Data Service (ANDS) nimmt im Bereich der Forschungsdaten eine absolut zentrale Stelle ein. ${ }^{28}$ ANDS profitiert von großzügiger staatlicher Förderung und hat zur Aufgabe, die bessere Nutzung von Forschungsoutput zu unterstützen, Wissenschaftler beim Datenmanagement $\mathrm{zu}$ helfen sowie neue und möglichst effiziente Forschung zu ermöglichen. ANDS stellt selber kein Datenarchiv für Forschungsdaten zur Verfügung, sondern versteht sich vielmehr als Online-Portal oder Schaltstelle. Die Fäden sämtlicher Projekte und Aktivitäten im

26 Vgl. hierzu: Mamtora, J.: Transforming Library Research Services: Towards a Collaborative Partnership. In: Library Management 34(4/5) (2013) S. 352-371 sowie Du, J. T.; Evans, N.: Academic Library Services Support for Research Information Seeking. In: Australian Academic \& Research Libraries 42(2) (2011) S. 103-120.

27 Vgl. hierzu Steiner, A.; Thomas, J.A.; Thompson, E.E.: Supporting Research at QUT: A Tale of Three Librarians and a Creative Industries Super-Faculty. In: Arts Library Society Australia \& New Zealand Conference (ARLIS/ANZ 2012), 6-8 September 2012. National Gallery of Victoria, Melbourne. Online zugänglich: http://eprints.qut.edu.au/54 208.

28 ANDS wird von der Regierung Australiens unterstützt. Die Verantwortung für den Service liegt allerdings beim National Collaborative Research Infrastructure Strategy Program und der Super Science Initiative des Education Investment Fund (EIF). 
Bereich Forschungsdatenmanagement fließen in ANDS zusammen.

Die großen australischen Forschungsförderer haben bisher kein Mandat für die Open-Access-Veröffentlichung von Forschungsdaten erteilt. Maßgeblich ist der Australian Code for the Responsible Conduct of Research aus dem Jahr 2007, der sukzessive um neue Paragraphen erweitert wird. So verlangt der Australian Research Council (ARC) ab 2014 für neue Projekte ein Datenmanagementplan und für die Speicherung von Forschungsdaten wird ein öffentlich zugängliches Repositorium dringend empfohlen. ARC setzt hiermit ein unmissverständliches Signal, verzichtet allerdings bewusst auf ein Open-Access-Mandat für Forschungsdaten, da man die unterschiedlichen Kulturen und Praktiken der verschiedenen Einrichtungen und Disziplinen respektieren möchte. ${ }^{29}$ Es liegt nun im Verantwortungsbereich der verschiedenen Universitäten, Richtlinien für die Erstellung von Datenmanagementplänen zu entwickeln und technische Infrastrukturen für Metadaten und Forschungsdaten zur Verfügung zu stellen.

ANDS bietet über das Online-Portal Research Data Australia $^{30}$ ein zentrales Verzeichnis aller Schlüsselpersonen, Aktivitäten, Institutionen, Metadaten und Forschungsdaten an. Darüber hinaus stellt ANDS auch themenspezifische Ratgeber, Weiterbildungsmaterial, Webinars, technische Dokumentationen, etc. zur Verfügung. Ein Netz von Partnerschaften mit verschiedenen Forschungsförderern stellt sicher, dass unterschiedliche und fachspezifische Bedürfnisse berücksichtigt, Teilprojekte initiiert und finanziert, Expertenwissen genutzt und Kreativität gezielt gefördert werden. ANDS ist außerdem zuständig für die Vergabe von DOIs: eine Voraussetzung für die korrekteZitierung von Datensets. ${ }^{31}$

Was ANDS nicht anbietet, ist der eigentliche Speicherplatz für Forschungsdaten. Hier verweist ANDS auf die institutionellen Repositorien der einzelnen Universitäten, auf internationale Fachrepositorien, auf die Cloud-basierte Lösung NeCTAR (National eResearch Collaboration Tools and Resources) oder das Projekt Research Data Storage Infrastructure (RDSI). ${ }^{32}$

29 Laughlin, Greg: Data in Spotlight under ARC Changes. In: Share. Newsletter of the newsletter of the Australian National Data Service. Issue 18 (April 2014) S. 2. Online zugänglich: http://ands.org.au/new sletters/share_issue18.pdf.

30 Research Data Australia: http://researchdata.ands.org.au/.

31 Beispiel des Zitats eines Datensets: Hanigan, Ivan: Meteorological Data for Australian Postal Areas. Australian Data Archive. 2010. DOI: 10.4225/13/50BBFCFE08A12RDA.

32 ANDS and Data Storage: http://www.ands.org.au/guides/sto rage.html.
Die meisten Bibliotheken kamen erstmals über das Rahmenprojekt „Seeding the Commons“ in Kontakt mit Forschungsdaten..$^{33}$ Bedingung für die Teilnahme am Projekt war das Durchführen von Interviews mit Wissenschaftlern zu ihrem Datenverhalten sowie das Erfassen einer bestimmten Anzahl an Metadatensätzen zu Forschungsdaten im zentralen ANDS-Verzeichnis. Beinahe alle Universitäten nahmen am Projekt teil und die Bibliothekare erzählen mit Begeisterung von den Dateninterviews und den neuen Kontakten zu Forschungsgruppen und verwandten Servicestellen an der Universität. ${ }^{34}$ Das Programm „Seeding the Commons“ diente sozusagen als Startschuss; nun sind die Universitäten gefordert, eine eigene Strategie für Forschungsdaten zu entwickeln. Gespräche an verschiedenen Hochschulen zeigen, dass die Bibliothekare stark involviert sind im Prozess der Rollenfindung, das heißt in der Diskussion der Verteilung der Verantwortlichkeiten zwischen Bibliothek, IT-Diensten, Research Office und Forschungsgruppen. Im Zentrum der Diskussionen stehen Verantwortlichkeiten für Datensammelpolitik, Metadatenmanagement, Richtlinien und Hilfestellung zu Datenmanagementplänen, Datenspeicherung, Zugang zu den Daten, rechtliche und ethische Fragestellungen, langfristige Archivierung, usw.

Eine Recherche im Zentralregister Research Data Australia $^{35}$ zeigt, wie erwartet, die große Heterogenität von Forschungsdaten: Hier findet man alles vom Abattoir regulation book 1895-1915 (State Records NSW) bis hin zu den Zooplankton surveys of Malakula Island, Vanuatu (Australian Institute of Marine Science). Manche Datensets sind frei aufrufbar - sofern man über das richtige Softwareprogramm verfügt -, andere sind nur auf Anfrage zugänglich oder gesperrt. Einige umfassen ein riesiges Datenvolumen, bei anderen findet man lediglich einen A4-Fragebogen, der zur Wiederverwendung frei zur Verfügung gestellt wird. Der einfache und einheitliche Zugang zu den Metadaten über das gemeinsame Portal täuscht über die Vielfalt der Datenquellen, die technischen Hürden zur Datennutzung sowie die rechtlichen und ethischen Fragestellungen bei der Nutzung oder Wiederverwendung von Forschungsdaten hinweg. Nichtsdestotrotz gehört Austra-

33 „Seeding the Commons“ kann sinngemäß als „Begrünung der Allmende“ übersetzt werden.

34 Vgl. hierzu: Bradley, L.: ANU Library‘s Support for Research Data Management. In: Incite 34(4) (2013) S. 26 oder Norman, B.; Stanton, K.V.: From Project to Strategic Vision: Taking the Lead in Research Data Management Support at the University of Sydney Library (2014, noch nicht veröffentlicht).

35 Research Data Australia: http://researchdata.ands.org.au/. 
lien im Bereich des Forschungsdatenmanagements zweifelsohne zu den weltweit führenden Nationen.

\section{Verankerung forschungsunter- stützender Services in der Bibliotheken}

Alle oben aufgeführten Services sind ohne Zweifel sinnvoll und nützlich für die Forschungsunterstützung, aber woher kommen die Ressourcen zur Bewältigung dieser neuen Aufgaben? Und wie sind sie in der Bibliothek organisatorisch verankert?

Während für den Aufbau von institutionellen Repositorien und für Projekte im Bereich Forschungsdatenmanagement - zumindest in der Anfangsphase - nationale Fördermittel zur Verfügung standen oder stehen, müssen alle anderen oben genannten Serviceleistungen über den regulären Etat geleistet werden. Australische Bibliotheken sehen sich also zunehmend gezwungen, Einsparungen in anderen Bereichen vorzunehmen, um diese neuen Aufgaben zu stemmen.

Hier sieht man sich als Teil eines größeren Trends, der v.a. in den angloamerikanischen Bibliotheken verbreitet ist und für ein generelles Überdenken und eine Neuausrichtung der Serviceleistungen plädiert. Ziel ist die Erreichung einer besseren Ausgewogenheit der Bibliotheksleistungen für die Forschung versus der Lehre. ${ }^{36}$ So herrscht unter diesen Autoren die Erkenntnis oder Meinung, dass der Wechsel hin zur elektronischen Informationsversorgung die Bibliothek für viele Wissenschaftler nahezu unsichtbar gemacht hat. Von den neuen „sichtbaren“ Dienstleistungen der Bibliothek profitieren stattdessen vor allem die Studierenden. $\mathrm{Zu}$ diesen neuen Services gehören insbesondere die Vermittlung von Informationskompetenz, aber auch die Integration von Bibliotheksressourcen in E-Learning, Computerarbeitsplätze, Gruppenarbeitsräume, längere Öffnungszeiten, Online-Chat-Services usw.

Selbstverständlich profitiert die Forschung stark vom E-Journal oder Datenbankangebot einer Bibliothek, aber die moderne, kundenorientierte Bibliothek will sich nach Ansicht vieler australischer Autoren nicht primär über ihre

36 Vgl. hierzu beispielsweise: Corrall; Kennan; Afzal (Anm. 24) und Auckland, M.: Re-skilling for Research: An Investigation into the Roles and Skills of Subject and Liaison Librarians Required to Effectively Support the Evolving Information Needs of Researchers. London 2012. Online zugänglich: http://www.rluk.ac.uk/content/re-skilling-r esearch oder Richardson et al. (Anm. 25).
Sammlung identifizieren. ${ }^{37}$ Vielmehr möchte man sich als Partner im Publikationsprozess oder Forschungszyklus verstehen und einen konkreten Mehrwert für den Wissenschaftler schaffen. Die Bibliothek möchte nicht lediglich als Informationslieferant dienen, sondern aktiv zur Wissensgenerierung beitragen; eine Neuorientierung nach dem Motto: „From content collection to content creation“.

Wie eingangs erwähnt, interessieren an dieser Stelle zwei Fragestellungen. Erstens, woher kommen die Ressourcen zur Bewältigung dieser neuen Tätigkeiten? Und zweitens, wie sind diese Aufgaben in der Organisationsstruktur der Bibliothek verankert? Zur Beantwortung dieser Fragen wurde einerseits die australische Fachliteratur analysiert und andererseits Gespräche an verschiedenen australischen Universitäten geführt. ${ }^{38}$

Als Resultat dieser Studie wurden drei Lösungsansätze erkannt, die hier vorgestellt werden: (a) Verschlankung des Angebotes für Studierende; (b) Fokussierung der Tätigkeit von Liaison Librarians auf forschungsunterstützende Funktionen und Bildung von fachbezogenen Teams zur Nutzung von Synergien; (c) Schaffung neuer Positionen zur Forschungsunterstützung bzw. zur Koordination dieser Aufgaben. Als weitere Rationalisierungsmaßnahmen, die in Gesprächen erwähnt wurden, aber hier nicht weiter ausgeführt werden, gelten die gezielte Förderung der Automatisierung und das Outsourcen von Arbeiten ${ }^{39}$, die starke räumliche und organisatorische Zentralisierung des Bibliotheksbetriebs ${ }^{40}$ sowie die Definition einer Sammelpolitik basierend auf Bevorzugung des elektronischen Mediums ${ }^{41}$.

37 Hier muss man ergänzen, dass die Sammlungen an australischen Universitätsbibliotheken im Vergleich zu Europa sehr jung sind; bedeutende Spezialsammlungen findet man hier kaum.

38 Folgende Universitätsbibliotheken wurden im Mai/Juni 2014 besucht: Australian Catholic University, Macquarie University, University of New South Wales, University of Sydney, University of Western Sydney, Wollongong University.

39 Zwei wichtige Aufgabenbereiche deutscher wissenschaftlicher Bibliothekare sind in Australien bereits fast vollständig outgesourct: Die inhaltliche Erschließung wird von der Katalogisierungsabteilung geleistet bzw. von den Fremddaten übernommen. Die Literaturauswahl wird beinahe an allen australischen Universitäten von den Fakultäten und/oder über maßgeschneiderte „Approval Plans“ von den Bibliothekslieferanten geleistet.

Hinsichtlich der Medienbearbeitung besteht ein Trend zur Vergabe dieser Arbeiten an Bibliothekslieferanten; bei Print-Medien prüfen viele Bibliotheken die Einführung von „Shelf-ready“-Services.

40 Australische Universitätsbibliotheken sind durchgängig einschichtig organisiert; der Prozess der Zusammenführung von Instituts- oder Departmentsbibliotheken in die Zentralbibliothek wurde bereits vor Jahren begonnen und wird, wo geographisch möglich, konsequent weitergeführt.

41 In der Regel wird das elektronische Medium (Zeitschrift, Buch) bevorzugt. Ist eine elektronische Ausgabe nicht erhältlich, oder wün- 


\subsection{Verschlankung des Angebotes für Studierende}

Immer wieder liest und hört man die Klage, dass die Vermittlung von Informationskompetenz an Studierende zu viel Zeit beanspruchte: Liaison oder Subject Librarians verbrachten früher sehr viel Zeit mit der Planung, Vorbereitung und Durchführung von Schulungen dieser Art. Die anfängliche Begeisterung für das Thema ist inzwischen etwas abgeschwächt - zumindest auf Ebene der wissenschaftlichen Bibliothekare - und eine Direktorin meinte sogar: „Ich kann das Wort Informationskompetenz nicht mehr hören!“ Gleichzeitig bezweifelt niemand die Notwendigkeit solcher Maßnahmen. Es geht also darum, die Informationskompetenzvermittlung möglichst schlank, effizient und effektiv zu gestalten.

Um die große Anzahl von Studierenden im Grundstudium möglichst ressourcenschonend $\mathrm{zu}$ schulen, sollen verstärkt oder ausschließlich Online-Tutorials eingesetzt werden. Kooperationen mit verwandten universitären Servicezentren für Lehre und Studium, für akademische Fähigkeiten (academic skills), akademische Integrität u.ä. sollen sicherstellen, dass die Online-Tutorials möglichst umfassend und nutzbringend sind. Ziel ist, dass die Tutorials in die studentische Lehrplattform eingebunden und die Studierenden verpflichtet werden, die Module durchzuarbeiten. Statt eines flächendeckenden Pflichtseminars zu Beginn des Semesters sollen die Module genau zum richtigen Zeitpunkt (just-in-time) und bedarfsorientiert in den Unterricht eingebunden werden. Die Verantwortung für die Vermittlung von Informationskompetenz auf Ebene des Grundstudiums wird in der Regel von der Benutzungsabteilungen geleistet, so dass Liaison Librarians von diesen Aufgaben vollständig befreit sind.

Auch andere Angebote für Studierende wurden verschlankt oder vollständig automatisiert. Beispielhaft zu nennen sind die Selbstausleihe und -rückgabe, die Erweiterung des E-Book-Angebotes, die Reduktion der Anzahl von Servicestellen und Informationstheken, der Abbau von Lehrbuchsammlungen zugunsten elektronischer Studienapparate, die Online-Buchung von Gruppenräumen, die Erweiterung der Online-Hilfestellungen über FAQs, Zusammenlegung von Bibliotheken auf dem Campus, usw. ${ }^{42}$ Einige Bibliothekare verrieten mir, dass sie anfangs Skru-

schen die Benutzer eine gedruckte Ausgabe, so wird die Printausgabe erworben. E-Book-Käufe über Paketlösungen und Patron-DrivenAcquisitions (PDA) sind weit verbreitet.

42 Vgl. hierzu beispielsweise: Sparks, J. et al.: Embedding Innovation for Scholarly Information and Research. In: Library Management 34 (1-2) (2013) S. 128-140 und Drummond; Wartho (Anm. 23). pel hatten, den Studierenden so viele Leistungen über Selbstbedienung oder Selbsthilfe anzubieten. Aber inzwischen ist Self-Service Teil des modernen Lebens und man stört sich nicht mehr daran.

\subsection{Fokussierung der Tätigkeit von Liaison Librarians auf forschungsunterstützende Aufgaben und Bildung von fachbezoge- nen Teams zur Nutzung von Synergien}

Die Bezeichnung Liaison oder Faculty Librarian ist nicht neu; der Begriff wurde in angloamerikanischen Bibliotheken bereits in den Neunzigerjahren eingeführt. ${ }^{43} \mathrm{Neu}$ ist vielmehr die verstärkte Ausrichtung dieser Fachkräfte auf die Forschungsunterstützung. An einigen Bibliotheken werden sie entsprechend auch Research Librarians genannt.

Während die Liaison Librarians in Australien früher sehr selbständig und eigenverantwortlich gearbeitet haben, bevorzugt man heute einen teambezogenen Ansatz. Diese Organisationsform hat aus Sicht der Bibliotheksleitung mehrere Vorteile: Sicherstellung guter Kommunikation zwischen den verschiedenen Liaison Librarians, Vermeidung des „single point of failure“ bei Ausfall eines Mitarbeiters, bessere Verteilung der Arbeitslast zwischen Mitarbeitern, Gewährleistung eines einheitlichen ServiceLevels und optimale Nutzung von gemeinsamen Tools.

Das neue Stellenprofil eines Liaison Librarians unterscheidet sich signifikant aber nicht radikal vom früheren Ansatz. Sie sind weiterhin zuständig für spezifische oder komplexere Fachrecherchen und -beratungen, werden allerdings darüber hinaus aufgefordert, Hilfestellung im Bereich Research Impact anzubieten, aktiv Kontakte zu (neuen) Wissenschaftlern aufzunehmen, in forschungsspezifischen Arbeitsgruppen/Kommissionen der Universität mitzuwirken und als Schnittstelle zum institutionellen Repositorium oder Forschungsdatenmanagement zu agieren. Damit Liaison Librarians ihr neues, erweitertes Aufgabenportfolio erfüllen können, werden sie meist vom

43 In diesem Aufsatz wird „Liaison Librarian“ als Bezeichnung für das Äquivalent eines Fachreferenten oder wissenschaftlichen Mitarbeiters in einer deutschsprachigen Bibliothek genutzt. Im Gegensatz zum deutschen Modell wird im anglo-amerikanischen Raum meist kein tiefes Fachwissen vorausgesetzt; gefordert werden vielmehr ein Bibliotheksstudium sowie ein grundsätzliches Interesse und Verständnis der Wissenschaftskommunikation in einem oder mehreren Fachgebieten. Vergleiche auch: Keller, Alice: „Subject Librarians“ in Großbritannien: Von der Bestands- zur Benutzerorientierung. In: BIBLIOTHEK - Forschung und Praxis 36 (1) (2012) S. 10-22. 
Dienst an der Informationstheke (früher 20-50\% ihrer Arbeitszeit), von der Informationskompetenzvermittlung für Studierende und von sammlungsspezifischen Aufgaben befreit.

Einige Bibliotheken unterscheiden zwischen Outreach Librarians, die aktiv (und zum Teil relativ aggressiv) und systematisch neue Kontakte zu Forschern herstellen, und Academic Service Librarians, die eher im Hintergrund agieren und aufwendigere Recherche- oder Beratungsaufgaben übernehmen. ${ }^{44}$ Diese Zweiteilung des Personal könnte auch dahingehend gedeutet werden, dass wissenschaftliche Mitarbeiter unterschiedliche Fähigkeiten und Charaktereigenschaften haben.

Erfolgreiche Liaison Librarians nach dem neuen Modell besitzen ausgezeichnete Kommunikationskompetenzen, gute analytische Fähigkeiten und schnelle Auffassungsgabe. Außerdem müssen sie die strategischen Forschungsprioritäten und Leistungsindikatoren der Universität kennen. Allgemein besteht der Wunsch, dass der Beitrag von Liaison Librarians zum Erfolg der Universität klar erkennbar und möglichst quantifizierbar ist. ${ }^{45}$ Allerdings hat man hierzu noch keine allgemeingültigen, geeigneten Indikatoren gefunden. In diese Richtung zielen allerdings die Customer Relationship Management (CRM)ähnlichen Systeme, die an manchen Bibliotheken eingesetzt werden und ein regelmäßiges Controlling der Kontakte und Leistungen ermöglichen.

\subsection{Schaffung neuer Positionen zur Forschungsunterstützung bzw. zur Koordination dieser Aufgaben}

Trotz der Erweiterung des Aufgabenportfolios der Liaison Librarians, ist es weder möglich noch sinnvoll, alle neuen Services in diese Stellenbeschreibungen zu packen. Man findet an australischen Bibliotheken drei Arten von neuen Positionen oder Organisationseinheiten, die zur Forschungsunterstützung beitragen: (1) Manager für das institutionelle Repositorium, (2) Spezialisten für Forschungsdatenmanagement und/oder eScholarship sowie (3) Fachpersonen zur Koordination für alle forschungsunterstützenden Aktivitäten.

Für den Aufbau von Repositorien wurde ein nationales Rahmenprojekt lanciert, so dass innerhalb relativ kurzer

44 Die Stellenbezeichnungen variieren je nach Bibliothek. Dieses Modell findet man beispielsweise an der University of New South Wales sowie University of Wollongong.

45 Siehe: Corrall; Kennan; Afzal (Anm. 24), Drummond; Wartho (Anm. 23), Richardson et al. (Anm. 25).
Zeit eine solide und koordinierte Infrastruktur an allen Universitäten aufgebaut werden konnte. ${ }^{46}$ Seit Ablauf dieser Förderphase sind die Bibliotheken nun selber verantwortlich für den Routinebetrieb dieser Systeme. Die Erfassung und Pflege von Metadaten sind in der Regel Aufgabe der Katalogisierungsabteilung - diese nennen sich neu Discovery Services o. ä.; für die technische und strategische Betreuung von Repositorien findet man in den meisten Organigrammen einen Repository Manager oder Repository Coordinator. Die notwendigen Einsparungen in den Abteilungen für Katalogisierung oder Medienbearbeitung werden durch die verstärkte Nutzung von Fremddaten und Dienstleistungen von Lieferanten (Approval Plan, Shelfready-Lieferung, Umstellung auf E-Book-Pakete) erwirkt. Das Führen der Repositorien fällt laut Burrows und Croker klar in den Verantwortungsbereich der Bibliotheken. ${ }^{47}$

Bei den Forschungsdaten hingegen ist die Rollenverteilung zwischen Bibliothek, IT-Diensten und Research Office noch weitgehend ungeklärt. Hier fließen derzeit über ANDS auch zentrale Fördermittel, so dass vielerorts Projektstellen finanziert werden können. ${ }^{48} \mathrm{Klar}$ ist, dass keine Universitätsabteilung im Alleingang den Gesamtkomplex zu Forschungsdaten bearbeiten und bedienen kann. ${ }^{49}$ Es braucht vielmehr ein Team an Spezialisten, die gemeinsam die erforderlichen Kenntnisse in den Bereichen technische Infrastruktur, Metadaten, rechtliche und ethische Rahmenbedingungen, Datenarchivierung usw. mitbringen. An einigen Universitäten findet man spezifische Abteilungen, die sich nebst anderen Aufgaben auch intensiver mit Forschungsdatenmanagement befassen; diese tragen in der Regel den Begriff eResearch oder eScholarship im Titel. eResearch-Abteilungen sind entweder den IT-Diensten angegliedert oder als eigenständige Servicezentren organisiert und bieten nebst Forschungsdatenarchivierung auch Visualisierungen, Hochleistungsrechner, virtuelle Laboratorien, usw..$^{50}$ An großen Biblio-

46 Siehe Kennan, M.A.; Kingsley, D.A.: The State of the Nation: A Snapshot of Australian Institutional Repositories. In: First Monday, Feb. 2009. Online zugänglich: http://pear.accc.uic.edu/ojs/index. $\mathrm{php} / \mathrm{fm} /$ article/view/2282/2092.

47 Burrows, T.; Croker, K.: Supporting Research in an Era of Data Deluge: Developing a New Service Portfolio within Information Services at the University of Western Australia. In: Paper presented at VALA 2012. Melbourne, February 6-9, 2012. Online zugänglich: http://www.vala.org.au/vala2012-proceedings/vala2012-session-1-bu rrows.

48 Vgl. hierzu Abschnitt 2.5.

$49 \mathrm{Vgl}$. hierzu beispielsweise Richardson et al. (Anm. 25), Corrall; Kennan; Afzal (Anm. 24).

50 Beispiel Monash eResearch Centre: https://platforms.monash.ed u/eresearch/. Burrows; Croker (Anm. 47) erkennen allerdings, dass 
theken findet man gelegentlich Abteilungen zu eScholarship (Sydney eScholarship, Melbourne eScholarship Research Centre ${ }^{51}$ ), die ursprünglich für die Digitalisierung von Bibliotheksbeständen und zur Langzeitdatenerhaltung gebildet wurden und sich nun zunehmend auch den Forschungsdaten widmen. An den meisten Universitäten wird das Thema Forschungsdaten allerdings in abteilungsübergreifenden Arbeitsgruppen bearbeitet, in denen die Bibliothek stark beteiligt ist. ${ }^{52}$ Im Rahmen solcher abteilungsübergreifenden Kooperation scheint klar zu sein, dass die Bibliothek die Metadatenerfassung und -verwaltung übernehmen wird; eine Arbeit, die in der Regel von Data Librarians geleistet wird. ${ }^{53}$ Gespräche mit Bibliothekaren weisen auch darauf hin, dass Bibliotheken einen wesentlichen Beitrag in den Bereichen Projektmanagement, Datenmanagementplan, Beratung zu Urheberrechtsfragen ${ }^{54}$ sowie Prozessorganisation leisten können und möchten.

Die Vielfalt und Komplexität der forschungsunterstützenden Services einer australischen Universitätsbibliothek sind beeindruckend. Entsprechend erstaunt es nicht, dass manche Bibliotheken Koordinationsbedarf sehen und die Stelle eines Research Support Coordinators - oder ähnlich geschaffen haben..$^{55}$ Diese Personen verstehen sich als Schaltstelle zwischen den eher technisch orientierten Repository- oder eScholarship-Teams und den fachlich-inhaltlich orientierten Liaison Librarians. Zudem gelten sie meist als Spezialisten für Bibliometrie und als Anlaufstelle und Fürsprecher für Open Access. In ihrer Koordinationsrolle sind sie für die Kommunikation zwischen den Abteilungen, für die Weiterbildung von Liaison Librarians (z.B. Research Impact, Forschungsdaten) sowie für die strategische Planung von forschungsunterstützenden Services zuständig. Eine Fragestellung, mit der sie sich gegenwärtig vielerorts beschäftigen, ist die Konzeption und Einführung

der Begriff e-Research in Australien sehr unterschiedlich genutzt und verstanden werden kann.

51 Sydney eScholarship: http://sydney.edu.au/escholarship/; eScholarship Research Centre, Melbourne: http://www.esrc.unimelb.ed u.au/. Da nur sehr wenige australische Bibliotheken über bedeutende Spezialsammlungen verfügen, kommt der Digitalisierung an Universitätsbibliotheken vergleichsweise wenig Bedeutung zu.

52 Vgl. hierzu beispielsweise Norman; Stanton (Anm. 34) oder Bradley (Anm. 34)

53 Thomas, J.: Future-Proofing: The Academic Library's Role in eResearch Support. In: Library Management 32(1-2) (2011) S. 37-47.

54 An vielen australischen Universitäten verstehen sich die Bibliothekare als Spezialisten zu Urheber- und Lizenzrecht.

55 Beispiele: University of Western Sydney, Charles Darwin University (NTU), Queensland University of Technology, Swinburne University of Technology. Die genauen Stellenbezeichnungen variieren, möglich sind auch Research Librarian, Research Support Officer. von Datenmanagementplänen, die neuerdings von den Forschungsförderern verlangt werden. Zur erfolgreichen Ausführung ihrer Aufgaben sind die Research Support Coordinators, die von Mamtora als "discipline agnostic“ bezeichnet werden, stark abhängig von den Kontakten und Fachkenntnissen der Liaison Librarians. ${ }^{56}$

Research Support Coordinators gibt es an einigen, aber keineswegs an allen forschungsintensiven Universitäten. Aus den Gesprächen konnte ich nicht klar erkennen, ob es sich bei diesen Stellen eher um provisorische Übergangslösungen handelt (bis die Forschungsunterstützung an den Universitätsbibliotheken fest verankert ist) oder ob es auch langfristig einen Bedarf für solche Koordinatoren gibt. Mein persönlicher Eindruck ist, dass der Koordinationsbedarf von forschungsunterstützenden Services mit der Einführung des Forschungsdatenmanagements signifikant steigt, insbesondere vor dem Hintergrund, dass diese Aufgabe eine enge Zusammenarbeit verschiedener Universitätsabteilungen und -kommissionen erfordert. Für den langfristigen Bedarf solcher Stellen sprechen auch die Umfrageergebnisse von Corrall, Kennan und Afzal, die klar darauf hinweisen, dass das größte Hindernis zum Ausbau von Services rund um Bibliometrie und Forschungsdaten die fehlenden Kenntnisse, Fähigkeiten und das mangelhafte Selbstvertrauen von Bibliothekaren sind. ${ }^{57}$ Und genau hier, nämlich in der bibliotheksinternen Weiterbildung und Kommunikation, können die Research Support Coordinators eine entscheidende Rolle spielen.

Tabelle 2 versucht schematisch die Tätigkeitsfelder von Liaison Librarians, Repository Managers/Data Librarians, Research Support Coordinators und eScholarship/ eResearch Teams zu umreißen. Diese Stellen werden an den verschiedenen Universitäten unterschiedlich benannt und definiert. Entsprechend ist es nicht möglich, eine für alle Bibliotheken gültige Gliederung zu finden. Die Tabelle versucht vielmehr, einen Überblick über die verschiedenen am häufigsten vorkommenden Optionen zu geben. Ein Kreuz an zwei Stellen bedeutet, dass die Aufgabe von unterschiedlichen Stellen ausgeführt werden kann.

\section{Schlussfolgerung}

Forschungsunterstützung ist eine relativ neue aber strategisch zunehmend wichtige Aufgabe von australischen Universitätsbibliotheken. In einer Studie aus dem Jahr 2006 beschrieben Genoni, Merrick und Willson die Ver-

56 Vgl. Mamtora (Anm. 26) S. 359.

57 Siehe: Corrall; Kennan; Afzal (Anm. 24). 
Tab. 2: Schematische Darstellung der Tätigkeitsfelder verschiedener Stellen in der Forschungsunterstützung. (Selbstverständlich gibt es lokale Unterschiede bzw. Ausnahmen.)

\begin{tabular}{|c|c|c|c|c|}
\hline & Liaison Librarians & $\begin{array}{l}\text { Repository Managers/ } \\
\text { Data Librarians }\end{array}$ & $\begin{array}{l}\text { Research Support } \\
\text { Coordinators }\end{array}$ & $\begin{array}{l}\text { eScholarship/ } \\
\text { eResearch Teams }\end{array}$ \\
\hline Collection Development & $\begin{array}{l}\text { No longer part of job } \\
\text { profile }\end{array}$ & & & \\
\hline Reference Work at Reference Desk & $\begin{array}{l}\text { No longer part of job } \\
\text { profile }\end{array}$ & & & \\
\hline Advanced Reference Queries & $\mathrm{x}$ & & & \\
\hline Faculty Liaison & $x$ & & & \\
\hline Advanced Information Literacy & $\mathrm{x}$ & & & \\
\hline Bibliographic management (Endnote et al.) & $x$ & & & \\
\hline Specific HDR Student Support & $x$ & & & \\
\hline Research Impact Cycle / Metrics & $\mathrm{x}$ & & $\mathrm{x}$ & \\
\hline Copyright \& Open Access & $x$ & & $x$ & \\
\hline $\begin{array}{l}\text { Scholarly Communication / } \\
\text { Scholarly Publishing Lifecycle }\end{array}$ & $x$ & & $\mathrm{x}$ & \\
\hline Institutional Repository & & $\mathrm{x}$ & & \\
\hline Digitization \& Data Archiving & & $\mathrm{x}$ & & $\mathrm{x}$ \\
\hline Research Data Management & & $\mathrm{x}$ & $\mathrm{x}$ & $\mathrm{x}$ \\
\hline eResearch & & & $x$ & $x$ \\
\hline
\end{tabular}

änderungen in der Wissenschaftskommunikation und empfahlen, dass die Bibliotheken ihre Services zukünftig stärker auf die Forschung ausrichten sollten. ${ }^{58}$ Im gleichen Jahr fand an der University of New South Wales die erste Umstrukturierung zugunsten der Forschungsunterstützung statt. ${ }^{59}$ Die Einführung der Leistungsevaluationen ERA (vormals RQF) im Jahr 2007 gab einen weiteren Impuls für die Stärkung der Ausrichtung auf die Forschung. Inzwischen gehört die Forschungsunterstützung $\mathrm{zu}$ den wichtigen strategischen Prioritäten aller australischen Universitätsbibliotheken. Parker schreibt hierzu: „Teaching and learning are our bread and butter, but the strategic directions of most Australian universities revolve around strengthening their position in research."60

Die australischen Bibliothekare sind in der Regel stolz auf ihre Leistungen im Bereich der Forschungsunterstützung. Gerne weisen sie darauf hin, wie der Beitrag ihrer Bibliothek erfolgreiche Forschungsanträge konkret unter-

58 Siehe Genoni, P.; Merrick, H.; Willson, M.A.: Scholarly Communities, e-Research Literacy and the Academic Librarian. In: The Electronic Library 24(6) (2006) S. 734-746.

59 Siehe: Drummond; Wartho (Anm. 23).

60 Parker (Anm. 1) S. 1. stützt hat. Immer wieder hört man die Meinung, dass die Bibliothek hierdurch ihren „kudos“, d.h. ihr Ansehen oder Renommee, innerhalb der Universität erhöhen konnte.

Das gegenwärtige Modell der Forschungsunterstützung durch die Bibliotheken wird stark gefördert von der Politik der Universitätsleitung bzw. der Regierung. Inwiefern klassisch bibliothekarische Werte oder Prioritäten diesen hochschulpolitischen Bedürfnissen untergeordnet werden, ist nicht klar. Die Rationalisierung von studentischen Angeboten zugunsten der Forschungsunterstützung wird von Bibliothekaren gelegentlich leicht bedauert, aber nicht grundsätzlich in Frage gestellt. Die faktische Umnutzung von institutionellen Repositorien zur Unterstützung der Leistungsevaluationen HERDC und ERA statt zur Förderung des Open-Access-Gedankens wird in der Regel von der Bibliotheksleitung als sinnvoller und unumgänglicher Schritt gesehen. ${ }^{61}$ Als ich leitenden Bibliothekaren die Frage nach dem Ausmaß der Selbst- versus Fremdbestimmung bei der Prioritätensetzung stellte, stieß ich vielerorts auf Unverständnis. Eine Bibliothekarin fasste ihre Meinung wie folgt zusammen: „The Library is the way we work

61 Vgl. hierzu beispielsweise Daly; McIntosh (Anm. 15). 
for the university.“ Das Wort „alignment“, also Angleichung der eigenen Zielsetzung an die der Universität, findet man häufig in der Literatur als Kennzeichen einer erfolgreichen Bibliotheksstrategie. Frägt man Bibliotheksdirektoren nach den Alleinstellungsmerkmalen ihrer Bibliotheken, so antworten sie oft mit den herausragenden Eigenschaften ihrer Universität.

In der Tat können sich die wenigsten Universitätsbibliotheken Australiens über ihre Sammlungen auszeichnen und identifizieren. Längst hat die Sammlung seine Bedeutung als Herzstück der Bibliothek verloren: Einerseits fehlen die Altbestände und Unikate, andererseits hat die starke Ausrichtung auf das elektronischen Medium das Konzept einer Sammlung vollständig verwässert. Dem Begriff Kulturgut - ein Aspekt der Bibliotheksarbeit, der zumindest in Europa vielerorts sozusagen als universitätsübergeordnet gewertet werden kann - bin ich in keinem
Gespräch begegnet. Die australischen Universitätsbiblio theken als Ganzes können sich also nur über ihre Services und über die „professional skills“ ihrer Mitarbeiter, nicht über die kulturelle Bedeutung ihres Bestands profilieren. Vor diesem Hintergrund erstaunt es nicht, dass die aktuelle Forschungsunterstützung einen so hohen Stellenwert besitzt.

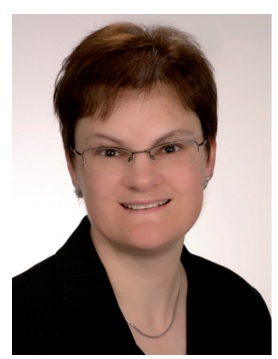

\section{Dr. Alice Keller}

Chefbibliothekarin Fachreferate /

Sacherschließung

Zentralbibliothek Zürich

Zähringerplatz 6

CH-8001 Zürich

Schweiz

alice.keller@zb.uzh.ch 